\title{
Complexo industrial e portuário do Pecém: um inquérito epidemiológico
}

\author{
Port and industrial compound of Pecém: an epidemiological survey
}

\author{
Sharmênia de Araújo Soares Nuto (https://orcid.org/0000-0002-4763-6773) ${ }^{1}$ \\ Edenilo Baltazar Barreira Filho (https://orcid.org/0000-0002-7641-677X) ${ }^{2}$ \\ Beatriz Fátima Alves de Oliveira (https://orcid.org/0000-0003-0103-3309) ${ }^{3}$ \\ Roberto Wagner Júnior Freire de Freitas (https://orcid.org/0000-0001-9295-1177) ${ }^{1}$ \\ Lucas de Oliveira do Couto (https://orcid.org/0000-0002-3758-3567) ${ }^{4}$ \\ Ludmilla da Silva Viana Jacobson (https://orcid.org/0000-0002-6698-4431) ${ }^{5}$ \\ Sandra de Souza Hacon (https://orcid.org/0000-0002-8222-0992) ${ }^{6}$ \\ André Reynaldo Santos Périssé (https://orcid.org/0000-0002-5253-5774) ${ }^{7}$
}

\footnotetext{
${ }^{1}$ Escritório Ceará, Fundação Oswaldo Cruz (Fiocruz). R. São José s/n, Precabura. 61760-000 Eusébio CE Brasil. shanuto@gmail.com ${ }^{2}$ Secretaria Executiva de Vigilância e Regulação em Saúde, Secretaria da Saúde do Estado do Ceará. Fortaleza CE Brasil.

${ }^{3}$ Escritório Piauí, Fiocruz. Teresina PI Brasil.

${ }^{4}$ Programa de Saúde Pública e Meio Ambiente, Escola Nacional de Saúde Pública (ENSP), Fiocruz. Rio de Janeiro RJ Brasil. ${ }^{5}$ Departamento de Estatística, Universidade Federal Fluminense. Niterói RJ Brasil.

${ }^{6}$ Departamento de Endemias, ENSP, Fiocruz. Rio de Janeiro RJ Brasil. ${ }^{7}$ Departamento de Ciências Biológicas, Fiocruz. Rio de Janeiro RJ Brasil.
}

\begin{abstract}
This research aimed to analyze the dwellers' knowledge of the territories, Pecém Port and Industrial Compound (CIPP), the quality of life of the communities living in the vicinity of the enterprises, and the prevalence of hypertension and overweight. This is a home-based, epidemiological survey of four areas of the Family Health Strategy (ESF) in São Gonçalo do Amarante and one area of the ESF in Caucaia, Ceará, Brazil. The study was carried out between 10/2017 and 03/2018. Anthropometric and blood pressure measurements were performed, and sociodemographic data and knowledge about community, the CIPP, and quality of life were collected. A total of $69.8 \%$ of the 503 adults interviewed were female, and the mean age was 44 years. Parada was the area reporting the highest percentage of problems related to air particles (51.1\%), the worst concept regarding the CIPP ( $40.1 \%$ bad), the highest percentage of worse quality of life $(29,1 \%)$, and greater desire to move to another place (31.5\%). Pecém had the highest percentage of community problems related to violence, consumption of illicit drugs, and prostitution. We concluded that there are signs of impact on local populations' health and quality of life without more significant direct work/income benefits.
\end{abstract}

Key words Environmental Health, Health Impact Assessment, Epidemiological Surveys
Resumo Esta pesquisa busca analisar o conhecimento dos moradores sobre o território, Complexo Industrial e Portuário do Pecém (CIPP), qualidade de vida das comunidades que vivem no entorno dos empreendimentos, além da prevalência de hipertensão e excesso de peso. Trata-se de um inquérito epidemiológico, de base domiciliar, envolvendo quatro áreas da Estratégia de Saúde da Família (ESF) do Município de São Gonçalo do Amarante e uma de Caucaia, Ceará, Brasil. O estudo foi realizado entre 10/2017 e 03/2018, em que foram realizadas aferições antropométricas $e$ pressão arterial e coletados dados sociodemográficos e o conhecimento sobre comunidade, CIPP e qualidade de vida. Dos 503 adultos entrevistados, $69,8 \%$ eram do sexo feminino e a média de idade foi 44 anos. Parada foi a área que relatou o maior percentual de problemas relacionados às partículas aéreas (51,1\%), pior conceito em relação ao CIPP $(40,1 \%$ ruim), maior percentual de piora da qualidade de vida $(29,1 \%)$ e maior desejo de mudar (31,5\%). Pecém apresentou maior percentual de problemas na comunidade relacionados à violência, consumo de drogas ilícitas e prostituição. Conclui-se que se apresenta indícios de impacto na saúde e qualidade de vida das populações locais sem maiores beneficios diretos no trabalho/renda. Palavras-chave Saúde Ambiental, Avaliação do Impacto na Saúde, Inquéritos Epidemiológicos 


\section{Introdução}

A implantação do Complexo Industrial e Portuário do Pecém (CIPP) no estado do Ceará tem início em 1996, estando sob a responsabilidade do governo do Estado e em consonância com o Plano Brasil em Ação do Governo Federal. A implantação do CIPP segue a política estadual desenvolvimentista, em seguimento a uma política nacional dos anos 1960, resultando, a partir da década de 1990, na atração para o Ceará de mais de 600 novas indústrias ${ }^{1}$.

A área do CIPP situa-se nos municípios de Caucaia e São Gonçalo do Amarante, Ceará, tendo o seu Terminal Portuário sido instalado no distrito de Pecém, antiga vila de pescadores, no litoral oeste do estado, a 60 quilômetros da capital. A localidade do Pecém foi escolhida para a implantação do empreendimento em função de sua posição privilegiada em relação aos principais mercados mundiais (Comunidade Europeia e Estados Unidos), e de suas características geográficas ${ }^{2}$.

Do ponto de vista econômico, o CIPP tem como objetivo o escoamento da produção com a ampliação do mercado e a diversificação da pauta de exportações. Atualmente, fazem parte do CIPP o terminal portuário e uma zona de processamento de exportação (ZPE), usinas termelétricas, indústrias de siderurgia e metalurgia, cerâmica, cimento e outros ${ }^{2}$. Para a instalação dos diversos empreendimentos, diferentes comunidades tradicionais foram removidas de seus territórios e outras atraíram diversos trabalhadores para as novas oportunidades de emprego ${ }^{3}$.

Nos territórios escolhidos para a localização de grandes empreendimentos com potencial poluidor, a perspectiva da geração de emprego e renda em contextos sociais vulnerabilizados pela pobreza historicamente construída faz com que sejam percebidos apenas os impactos econômicos positivos advindos da instalação dessas indústrias ${ }^{4}$. Mas, na verdade, resultam em novos riscos de doenças e agravos à saúde, interferência na organização comunitária, gerando o acirramento de desigualdades sociais, impactos ambientais e políticas governamentais direcionadas ao favorecimento das grandes corporações ${ }^{3,5}$. Os benefícios são geralmente distribuídos de forma heterogênea entre os habitantes, gerando percepções distintas em relação aos empreendimentos.

As desapropriações e reassentamentos trazem sérias consequências para as comunidades, uma vez que os novos espaços e paisagens ocupados obrigam essas pessoas a mudarem suas práticas, hábitos diários e a cultura, o que pode repercutir de forma negativa em sua saúde, bem-estar e qualidade de vida. Outras consequências desse processo podem ser observadas na saúde em decorrência das modificações da qualidade do ar, da água e do solo por contaminantes provenientes da indústria, seja pelas atividades de obtenção de matéria prima (extrativismo), seja pela queima de combustíveis na produção e transporte, $o$ que acaba resultando, também, em alterações no perfil sociodemográfico, cultural e econômico da região $0^{1,3}$.

O estudo dos impactos na saúde humana decorrente de grandes empreendimentos auxilia na compreensão da carga de doenças associadas às atividades econômicas instaladas e em fase de instalação nos territórios ${ }^{6}$. Igualmente, apontam a necessidade da ampliação e da qualificação das redes de atenção em saúde para lidar com os agravos da população decorrentes do ambiente e do trabalho ${ }^{3}$.

As percepções das comunidades implicadas por problemas ambientais, que afetam a saúde e qualidade de vida, têm sido utilizadas para subsidiar os processos decisórios políticos? ${ }^{7}$. Quando se estuda os impactos ambientais na saúde humana é importante à inclusão da percepção da população e trabalhadores que vivem no entorno dos empreendimentos ${ }^{8,9}$. Di Giulio et al. ${ }^{10}$ reforça essa importância exemplificando que em poucos estudos brasileiros sobre a saúde e condições de trabalho de cortadores de cana de açúcar, nenhum considerou a percepção de risco dos indivíduos.

A partir do contato com as Secretarias de Saúde de Caucaia e São Gonçalo do Amarante, identificou-se uma lacuna de conhecimento em relação aos impactos na saúde da população que vivem no entorno do CIPP e dificuldades das equipes da Estratégia de Saúde da Família (ESF) em lidar com as mudanças ocorridas nos territórios: violência urbana, prostituição, uso de drogas, alteração no consumo alimentar, dentre outros. O inchaço repentino na população local de trabalhadores para o Terminal Portuário e demais empreendimentos dificulta as práticas da ESF como visitas domiciliares, pois um domicílio não é mais composto por famílias, mas por 10 , 15, 20 trabalhadores, que estão morando temporariamente na região.

Diante do exposto, fazem-se os seguintes questionamentos: Como está a saúde da população do entorno? Como percebem a vinda dos empreendimentos? Que mudanças ocorreram? $\mathrm{Na}$ tentativa de responder a tais questionamen- 
tos, preencher a lacuna do conhecimento existente, assim como, colaborar nos territórios da Estratégia de Saúde da Família este estudo foi proposto. Com o objetivo de analisar, através de inquérito epidemiológico, o conhecimento dos moradores sobre o território, CIPP, qualidade de vida das comunidades que vivem no entorno dos empreendimentos, além da prevalência de hipertensão e obesidade.

\section{Método}

Trata-se de um inquérito epidemiológico, com coleta de dados domiciliar, com a perspectiva de subsidiar a realização de Avaliação de Impacto à Saúde (AIS). A população do estudo foi a residente na área de entorno do CIPP e envolveu quatro áreas da Estratégia de Saúde da Família (ESF) do Município de São Gonçalo (Praia, Planalto, Parada, Acende-Candeia) e uma área da ESF de Caucaia (Matões). A amostragem foi feita por conglomerado e os domicílios foram selecionados de forma aleatória em cada comunidade. Todos os moradores dentro dos critérios de seleção eram elegíveis para participar do estudo.

Foram incluídos os domicílios sorteados que durante a visita dos pesquisadores contavam com um morador acima de 18 anos em condições de responder ao formulário. Neste caso todos os moradores da casa eram elegíveis para participação na pesquisa, independente da faixa etárias. Foram excluídos os domicílios que se encontravam fechados após três tentativas de contato ou que contavam com a presença de maiores de 18 anos com alguma deficiência física ou mental que impedisse a comunicação. Neste artigo serão analisados apenas os dados dos adultos.

Entre outubro de 2017 e março de 2018, oito pesquisadores treinados, e após o estudo piloto, entrevistaram os moradores em seus domicílios e realizaram aferições antropométricas (peso e altura) e pressão arterial. O questionário foi desenvolvido para coletar informações sociodemográficas (ex. idade, raça/cor e atividade profissional), percepção dos moradores em relação às comunidades de moradia (ex. principais problemas e seus responsáveis), percepção sobre o CIPP e qualidade de vida, neste caso com o uso do inventário de qualidade de vida Short Form Health Survey (SF36) nas dimensões percepção geral de saúde, limitações das atividades diárias, limitações de desempenho em decorrência de problemas emocionais e limitações de atividades sociais ${ }^{11}$ e perguntas sobre uso de álcool, drogas e depressão ${ }^{12}$. Foram utilizados aparelhos portáteis eletrônicos para a coleta dos dados e envio seguro da informação para um servidor. O questionário foi montado dentro da plataforma ODK que é um conjunto de ferramentas de código aberto que ajuda as organizações a coletar e gerenciar dados ${ }^{13}$.

As atividades de campo iniciaram com o mapeamento das comunidades, principalmente para definição dos seus limites territoriais. Em seguida, os domicílios foram selecionados de forma aleatória. Ao iniciar uma nova subárea os 14 primeiros domicílios eram utilizados para fazer um sorteio e um deles era selecionado para iniciar as entrevistas. As visitas continuaram sempre pelo lado direito da rua até o fim e depois pelo outro lado, de modo a fazer os dois lados da rua. Realizou-se a coleta percorrendo todas as ruas paralelas e em seguida as perpendiculares dentro das áreas em estudo.

$\mathrm{O}$ estudo foi feito nas áreas de abrangência das equipes da ESF de Praia e Planalto (Pecém), Parada, Acende Candeias e Matões. Estimava-se em 18.000 pessoas o número de moradores nas comunidades em 2017. No cálculo do tamanho da amostra foram considerados nível de confiança de $95 \%$, erro amostral de 5\% e proporção esperada de 0,5 . O cálculo da correção para população finita considerou 18.000 pessoas, tendo sido acrescentados $20 \%$ ao cálculo final para cobrir eventuais perdas nas entrevistas. Como o tipo de amostragem foi conglomerada, também foi incluído um efeito de desenho de 2,5. O efeito de desenho é calculado pela fórmula: $\mathrm{DEFF}=1+(\mathrm{M}-1)$ $\rho(M=$ tamanho do conglomerado; $\rho=$ coeficiente de correlação intraclasse). Neste estudo foram considerados os valores de $M=4$ e $\rho=0,5$. Dessa forma, o tamanho da amostra final foi definido em 1.140 pessoas, distribuídos em 384 domicílios (famílias da ESF). Para garantir uma distribuição proporcional dessa amostra entre as comunidades, o inquérito levou em conta o tamanho das comunidades de acordo com número de famílias cadastradas em cada equipe da ESF.

Embora a seleção dos domicílios da amostra tenha sido realizada com muito cuidado e seguindo um protocolo bem definido, durante a coleta dos dados houve muitas recusas entre os moradores dos domicílios principalmente entre aqueles do sexo masculino. Dessa forma, um erro sistemático de coleta foi observado segundo sexo e faixa etária, que pode ser explicado pela maior facilidade de encontrar em casa e de entrevistar mulheres, principalmente as donas de casa. Assim, embora seja uma amostra probabilística, mas devido a possíveis desvios nas estimativas 
em relação aos parâmetros populacionais, a expansão da amostra por meio de pesos amostrais não foi implementada.

Os dados foram analisados dentro de cada comunidade e as frequências comparadas de acordo com a variável estudada. Para variáveis categóricas, as comparações foram feitas utilizando-se o teste de Qui-quadrado e para variáveis contínuas a análise de variância (Anova). A avaliação dos resultados do SF36 foi realizada mediante a atribuição de escores para cada questão e posteriormente transformado em escala de $0-100$, em que zero é a pior qualidade de vida e cem a melhor ${ }^{14}$. Calculou-se a média, desvio padrão e mediana de cada dimensão, sendo cada uma analisada separadamente. O nível de significância adotado foi de 0,05 . As análises foram realizadas no programa Stata 13.0.

O projeto de pesquisa foi submetido e aprovado pelo Comitê de Ética em Pesquisa da Universidade de Fortaleza (UNIFOR). Todos os participantes assinaram o Termo de Consentimento Livre e Esclarecido (TCLE).

\section{Resultados}

Foram entrevistados 687 indivíduos $(72,3 \%$ da amostra prevista). Os dados aqui apresentados são referentes aos 503 maiores de 18 anos incluídos, sendo 205 (41\%) no Pecém, 127 (25,2\%) na Parada, 88 (17,5\%) em Matões e $83(16,5 \%)$ em Acende Candeia. A média geral de idade foi de 44 anos, com predomínio do gênero feminino $(351 / 69,8 \%)$ na amostra, não sendo observada variação significativa do gênero dentro das comunidades. A raça/cor predominantes foi a parda, mas sem diferença na distribuição pelas comunidades. Embora não significativa, a comunidade de Matões foi a que apresentou menor proporção de idosos (14,8\%). Enquanto Acende Candeia $(60,2 \%)$ e Parada $(66,1 \%)$ apresentaram predomínio da religião católica, Matões $(46,6 \%)$ e Pecém $(38,0 \%)$ apresentaram grande proporção de evangélicos. Acende Candeia foi a comunidade com maior número de agricultores $(24,1 \%)$ e todas apresentaram baixo número de pescadores, construtores e colaboradores com o CIPP. Acende Candeia foi também a comunidade com a maior proporção de moradores trabalhando por conta própria $(41,7 \%)$ e com carteira assinada (29,2\%), enquanto Parada apresentou o maior percentual de servidores públicos (23,2\%). Matões apresentou o maior percentual de moradores com Bolsa Família (23,9\%) (Tabela 1).
Dentre os entrevistados, 39 (47\%) dos entrevistados em Acende Candeia, 56 (44\%) em Parada e 35 (39,77\%) moram na comunidade desde que nasceram. O menor percentual foi encontrado em Pecém (57 - 27,8\%), tendo essa diferença, por comunidade, significância estatística $(\mathrm{p}=0,000)$.

Poluição do ar $(28,3 \%)$ e poeira $(22,8 \%)$ foram as maiores reclamações dos moradores de Parada (Tabela 2). Já violência foi mais relatada no Pecém $(31,7 \%)$. O município correspondente foi identificado como o maior responsável pelo problema relatado em Acende Candeia (39,0\%) e Matões (37,5\%), enquanto o CIPP foi reportado com maior frequência em Parada (36,4\%). Álcool e drogas ilícitas foram mais identificados como problemas em Matões (67,0 e 83,0\%, respectivamente) e no Pecém (62,0 e 84,9\%, respectivamente). Acende Candeia foi a comunidade que menos identificou a prostituição como um problema $(16,9 \%)$. Matões e Parada foram identificadas como pouco seguras em relação à violência.

Matões $(87,5 \%)$ e Pecém $(84,4 \%)$ foram as comunidades com melhor conceito em relação ao CIPP, enquanto Parada $(40,1 \%)$ foi a comunidade que pior avaliou o CIPP (Tabela 3). Reforçando a percepção negativa em relação ao CIPP, Parada foi a área pesquisada com menor percentual de melhoria do padrão de vida com a implantação do CIPP $(22,0 \%)$ e maior de piora $(29,1 \%)$ do mesmo. Foi também a comunidade com maior percentual para desejo de mudar do lugar $(31,5 \%)$, enquanto Matões obteve o menor desejo de mudança $(13,6 \%)$.

Em relação às medidas de qualidade de vida, apenas a dimensão "problemas emocionais" foi estatisticamente significativa e o Pecém apresentou a menor média (62) entre as comunidades (Tabela 4). Sobrepeso (33,7\%), obesidade $(44,5 \%)$ e hipertensão $(28,7)$ foram mais frequentes no Pecém.

\section{Discussão}

Os dados apresentados estão relacionados à quatro comunidades do entorno do CIPP, três em áreas próximas ao complexo e uma mais afastada. A comunidade do Pecém, em São Gonçalo do Amarante, é a mais urbanizada e a maior de todas, e nossos dados apontam a percepção dos problemas mais comuns, entre seus moradores, os indicadores semelhantes de áreas mais urbanizadas, como violência (31,7\%), álcool (62\%), drogas ilícitas (84,9\%) e prostituição (61\%). Foi, 
Tabela 1. Informações sociodemográficas, por comunidade. São Gonçalo do Amarante e Caucaia, Ceará, 2018.

\begin{tabular}{|c|c|c|c|c|c|}
\hline \multirow[t]{2}{*}{ Variável } & $\begin{array}{c}\text { Acende } \\
\text { Candeia }(n=83) \\
\end{array}$ & Matões $(n=88)$ & $\begin{array}{c}\text { Parada } \\
(n=127)\end{array}$ & $\begin{array}{l}\text { Pecém } \\
(n=205)\end{array}$ & \multirow[t]{2}{*}{ p-valor } \\
\hline & n $(\%)$ & n (\%) & n (\%) & n (\%) & \\
\hline Gênero & & & & & 0,129 \\
\hline Feminino & $49(59,0)$ & $65(74,0)$ & $90(71,0)$ & $147(71,7)$ & \\
\hline Masculino & $34(41,0)$ & $23(26,0)$ & $37(29,0)$ & $58(28,3)$ & \\
\hline Idade & & & & & 0,073 \\
\hline 15-29 (jovens) & $30(36,1)$ & $27(30,7)$ & $28(22,0)$ & $47(22,9)$ & \\
\hline 30-59 (adultos) & $34(41,0)$ & $48(54,5)$ & $66(52,0)$ & $116(56,6)$ & \\
\hline 60 ou mais (idosos) & $19(22,9)$ & $13(14,8)$ & $33(26,0)$ & $42(20,5)$ & \\
\hline Raça/cor & & & & & 0,218 \\
\hline Pardo & $66(79,5)$ & $63(71,6)$ & $105(82,6)$ & $148(72,7)$ & \\
\hline Branco & $12(14,5)$ & $14(15,9)$ & $18(14,2)$ & $33(16,1)$ & \\
\hline Negro & $4(4,8)$ & $10(11,4)$ & $4(3,2)$ & $19(9,3)$ & \\
\hline Outro & $1(1,2)$ & $1(1,1)$ & $0(0,0)$ & $5(1,9)$ & \\
\hline Estado civil & & & & & 0,416 \\
\hline Solteiro & $25(30,1)$ & $28(31,8)$ & $29(22,8)$ & $67(32,7)$ & \\
\hline Casado/morando junto & $50(60,3)$ & $49(55,7)$ & $85(66,9)$ & $118(57,6)$ & \\
\hline Viúvo & $5(6,0)$ & $5(5,7)$ & $11(8,7)$ & $13(6,3)$ & \\
\hline Separado ou divorciado & $3(3,6)$ & $6(6,8)$ & $2(1,6)$ & $7(3,4)$ & \\
\hline Religião & & & & & 0,011 \\
\hline Católica & $50(60,2)$ & $39(44,3)$ & $84(66,1)$ & $106(51,7)$ & \\
\hline Evangélica & $22(26,5)$ & $41(46,6)$ & $39(30,7)$ & $78(38,0)$ & \\
\hline Outra & $0(0,0)$ & $0(0,0)$ & $1(0,8)$ & $2(1,0)$ & \\
\hline Não tenho religião & $11(13,3)$ & $8(9,1)$ & $3(2,4)$ & $19(9,3)$ & \\
\hline Escolaridade & & & & & 0,738 \\
\hline Não sabe ler & $11(13,3)$ & $9(10,2)$ & $16(12,6)$ & $20(9,7)$ & \\
\hline Fundamental incompleto & $25(30,1)$ & $25(28,4)$ & $43(33,9)$ & $64(31,2)$ & \\
\hline Fundamental completo & $5(6,0)$ & $10(11,4)$ & $12(9,4)$ & $20(9,8)$ & \\
\hline Ensino médio incompleto & $12(14,5)$ & $8(9,1)$ & $8(6,3)$ & $20(9,8)$ & \\
\hline Ensino médio completo & $28(33,7)$ & $32(36,4)$ & $37(29,1)$ & $66(32,2)$ & \\
\hline Ensino superior & $2(2,4)$ & $4(4,5)$ & $11(8,7)$ & $15(7,3)$ & \\
\hline Atividade profissional & & & & & $<0,001$ \\
\hline Pescador & $0(0,0)$ & $0(0,0)$ & $1(0,8)$ & $3(1,5)$ & \\
\hline Construção civil & $0(0,0)$ & $6(6,8)$ & $0(0,0)$ & $4(1,9)$ & \\
\hline Agricultor & $20(24,1)$ & $11(12,5)$ & $13(10,2)$ & $6(2,9)$ & \\
\hline Comerciante & $0(0,0)$ & $0(0,0)$ & $4(3,2)$ & $5(2,4)$ & \\
\hline Colaborador CIPP & $9(10,8)$ & $3(3,4)$ & $1(0,8)$ & $2(1,0)$ & \\
\hline Aposentado & $13(15,7)$ & $16(18,2)$ & $23(18,1)$ & $30(14,6)$ & \\
\hline Do lar & $17(20,5)$ & $31(35,2)$ & $42(33,1)$ & $67(32,7)$ & \\
\hline Estudante & $5(6,0)$ & $2(2,3)$ & $5(3,9)$ & $3(1,5)$ & \\
\hline Sem atividade & $9(10,9)$ & $9(10,2)$ & $7(5,5)$ & $27(13,2)$ & \\
\hline Outro & $10(12,0)$ & $10(11,4)$ & $31(24,4)$ & $58(27,8)$ & \\
\hline
\end{tabular}

continua

ainda, a comunidade que apresentou o pior valor da dimensão de limitação de desempenho em decorrência de problemas emocionais $(62,2)$, aquela com maior proporção de obesos $(44,5 \%)$, indicando fatores que possam estar afetando a qualidade de vida da população local. Para a comunidade de Matões, no município de Caucaia, álcool (67\%), drogas ilícitas (83\%) e insegurança recente $(72,8 \%)$ foram apontados como problemas sociais. 
Tabela 1. Informações sociodemográficas, por comunidade. São Gonçalo do Amarante e Caucaia, Ceará, 2018.

\begin{tabular}{|c|c|c|c|c|c|}
\hline \multirow[t]{2}{*}{ Variável } & $\begin{array}{c}\text { Acende } \\
\text { Candeia }(n=83)\end{array}$ & Matões $(n=88)$ & $\begin{array}{c}\text { Parada } \\
(n=127)\end{array}$ & $\begin{array}{c}\text { Pecém } \\
(n=205)\end{array}$ & \multirow[t]{2}{*}{ p-valor } \\
\hline & $\mathrm{n}(\%)$ & n (\%) & n (\%) & n (\%) & \\
\hline Tipo de vínculo trabalhista ${ }^{a}$ & & & & & 0,004 \\
\hline Conta própria & $20(41,7)$ & $11(29,7)$ & $22(39,3)$ & $37(35,6)$ & \\
\hline Carteira assinada & $14(29,2)$ & $7(18,9)$ & $7(12,5)$ & $19(18,3)$ & \\
\hline Sem carteira assinada & $2(4,2)$ & $4(10,8)$ & $5(8,9)$ & $10(9,6)$ & \\
\hline Empregador & $0(0,0)$ & $1(2,7)$ & $0(0,0)$ & $0(0,0)$ & \\
\hline Servidor Público & $1(2,1)$ & $3(8,2)$ & $13(23,2)$ & $4(3,9)$ & \\
\hline Desempregado & $2(4,2)$ & $0(0,0)$ & $1(1,8)$ & $6(5,7)$ & \\
\hline Outro/não respondeu & $9(18,6)$ & $11(29,7)$ & $8(14,3)$ & $28(26,9)$ & \\
\hline Seguridade social & & & & & 0,439 \\
\hline Não & $47(56,6)$ & $50(56,8)$ & $72(56,7)$ & $121(59,3)$ & \\
\hline Bolsa família & $14(16,9)$ & $21(23,9)$ & $25(19,7)$ & $38(18,6)$ & \\
\hline Aposentadoria & $21(25,3)$ & $17(19,3)$ & $25(19,7)$ & $32(15,7)$ & \\
\hline Pensão & $0(0,0)$ & $0(0,0)$ & $2(1,6)$ & $8(3,9)$ & \\
\hline Outro & $1(1,2)$ & $0(0,0)$ & $3(2,3)$ & $2(1,0)$ & \\
\hline
\end{tabular}

${ }^{\mathrm{a}} \mathrm{N}=249$; excluindo aposentados, do lar e estudantes; $\mathrm{n}=48$ (AC), $\mathrm{n}=46(\mathrm{M}), \mathrm{n}=57$ (PA) e $\mathrm{n}=105$ (PE).

Fonte: Elaborado pelos autores.

Pecém e Matões são os dois territórios mais próximos ao Terminal Portuário, distanciandose apenas por cinco quilômetros, tendo sido afetados diretamente pela instalação deste na região. Colaborando com a percepção das comunidades de Pecém e Matões, em estudo realizado sobre o uso de drogas com 323 adolescentes do Pecém, $33(10,2 \%)$ dos adolescentes se identificam como consumidores de drogas lícitas e ilícitas, em que as lícitas mais consumidas são o álcool (28 - 84,8\%) e cigarro (9 - 27,3\%). Já os que declararam ser usuários de drogas ilícitas, a maconha é a mais consumida ( 15 - 45,5\%) e em seguida, a cola $(4-12,1 \%)^{15}$.

Essa realidade também foi encontrada em outros estudos após a implantação do Complexo Petroquímico do Rio de Janeiro (COMPERJ) ${ }^{16-18}$. No estudo em Porto das Caixas e Sambaetiba, localizados em Itaboraí-RJ, em todos os grupos focais e $60 \%$ dos informantes-chave nas entrevistas ponderaram que houve aumento do risco de agressões, violência sexual, desemprego, prostituição e assaltos após a implantação do COMPERJ $^{16}$. No município de Itaboraí e áreas do entorno do COMPERJ, identificaram como impactos psicossociais: desemprego; violência física e sexual; o aumento da criminalidade; e a elevação do consumo e tráfico de drogas. Esses relatos foram ponderados pela existência de ou- tros fatores que relacionam com a vinda de grandes empreendimentos, tais como, desordem urbana, favelização, deslocamento de mão de obra, aumento do contato de pessoas de fora da região com moradores locais, ausência de aplicação dos recursos em infraestrutura local ${ }^{17}$.

Já a comunidade de Parada, situada em parte na direção da pluma de dispersão da siderúrgica do CIPP, apresenta um padrão peculiar com grande número de trabalhadores do serviço público $(23,2 \%)$, aposentado $(18,1 \%)$ e trabalhadores do lar $(33,1 \%)$. Parada foi, entre as comunidades, aquela que mais relatou queixas relacionadas a qualidade do ar (poluição e poeira; $51,1 \%$ ), a que mais identificou o CIPP como o responsável pelo problema $(36,4 \%)$, que a vida piorou após o CIPP $(29,1 \%)$ e faz uma avaliação negativa do CIPP (ruim - 40,1\%). Essa área, devido à proximidade da siderúrgica e direção do vento, é a que mais sofre os efeitos diretos da poluição atmosférica no CIPP, por isso constatamos uma visão mais crítica em relação aos empreendimentos.

A percepção de risco varia de acordo com as alterações climáticas e a proximidade dos eventos, em que a população identifica maiores riscos quando no seu entorno apresenta mudanças de temperatura ao longo do tempo, ou são áreas propensas à desastres naturais, ou tem altas emissões de dióxido de carbono ${ }^{19}$. Desta forma, a rela- 
Tabela 2. Percepções sobre a comunidade. São Gonçalo do Amarante e Caucaia, Ceará, 2018.

\begin{tabular}{|c|c|c|c|c|c|}
\hline \multirow[t]{2}{*}{ Variável } & $\begin{array}{c}\text { Acende } \\
\text { Candeia }(n=83) \\
\end{array}$ & $\begin{array}{c}\text { Matões } \\
(\mathbf{n}=\mathbf{8 8})\end{array}$ & $\begin{array}{c}\text { Parada } \\
(\mathrm{n}=127)\end{array}$ & $\begin{array}{l}\text { Pecém } \\
(\mathrm{n}=205)\end{array}$ & \multirow[t]{2}{*}{ p-valor } \\
\hline & $\mathrm{n}(\%)$ & n (\%) & n (\%) & n (\%) & \\
\hline Principal problema na comunidade & & & & & $<0,001$ \\
\hline Não tem & $6(7,2)$ & $8(9,1)$ & $9(7,1)$ & $8(3,9)$ & \\
\hline Poluição do ar & $8(9,6)$ & $9(10,2)$ & $36(28,3)$ & $48(23,4)$ & \\
\hline Falta d'água & $12(14,5)$ & $4(4,5)$ & $1(0,8)$ & $4(2,0)$ & \\
\hline Mosquitos & $3(3,6)$ & $12(13,7)$ & $3(2,4)$ & $18(8,8)$ & \\
\hline Violência & $2(2,4)$ & $17(19,3)$ & $5(4,0)$ & $65(31,7)$ & \\
\hline Poeira & $13(15,6)$ & $19(21,6)$ & $29(22,8)$ & $31(15,1)$ & \\
\hline Outro & $39(76,1)$ & $19(21,6)$ & $44(34,6)$ & $31(15,1)$ & \\
\hline Responsável pelo problema ${ }^{\mathrm{a}}$ & & & & & $<0,001$ \\
\hline Estado & $3(3,9)$ & $7(8,8)$ & $16(13,6)$ & $29(14,7)$ & \\
\hline Município & $30(39,0)$ & $30(37,5)$ & $14(11,9)$ & $26(13,2)$ & \\
\hline CIPP (geral) & $14(18,2)$ & $2(2,5)$ & $43(36,4)$ & $41(20,8)$ & \\
\hline Outro/não respondeu & $30(38,9)$ & $41(51,2)$ & $45(38,1)$ & $101(51,3)$ & \\
\hline $\begin{array}{l}\text { Considera abuso de álcool um } \\
\text { problema na comunidade }\end{array}$ & & & & & 0,001 \\
\hline Não & $29(35,0)$ & $26(29,5)$ & $65(51,2)$ & $76(37,0)$ & \\
\hline Sim & $47(56,6)$ & $59(67,0)$ & $55(43,3)$ & $127(62,0)$ & \\
\hline Não soube responder & $7(8,4)$ & $3(3,5)$ & $7(5,5)$ & $2(1,0)$ & \\
\hline $\begin{array}{l}\text { Considera uso de drogas ilícitas um } \\
\text { problema na comunidade }\end{array}$ & & & & & $<0,001$ \\
\hline Não & $23(27,7)$ & $12(13,7)$ & $35(27,6)$ & $30(14,6)$ & \\
\hline Sim & $45(54,2)$ & $73(83,0)$ & $82(64,6)$ & $174(84,9)$ & \\
\hline Não soube responder & $15(18,1)$ & $3(3,3)$ & $10(7,8)$ & $1(0,5)$ & \\
\hline $\begin{array}{l}\text { Considera a prostituição um } \\
\text { problema na comunidade }\end{array}$ & & & & & $<0,001$ \\
\hline Não & $61(73,5)$ & $45(51,1)$ & $50(39,4)$ & $70(34,0)$ & \\
\hline Sim & $14(16,9)$ & $38(43,2)$ & $62(48,8)$ & $125(61,0)$ & \\
\hline Não soube responder & $8(9,6)$ & $5(5,7)$ & $15(11,8)$ & $10(5,0)$ & \\
\hline $\begin{array}{l}\text { Tem se sentido inseguro ou } \\
\text { com medo no último ano na } \\
\text { comunidade }\end{array}$ & & & & & $<0,001$ \\
\hline Sim (uma/poucas vezes) & $24(29,0)$ & $13(14,8)$ & $38(30,0)$ & $52(25,4)$ & \\
\hline Sim (várias vezes) & $29(35,0)$ & $51(58,0)$ & $52(41,0)$ & $51(24,8)$ & \\
\hline Não & $30(36,0)$ & $24(27,2)$ & $37(29,0)$ & $102(49,8)$ & \\
\hline $\begin{array}{l}\text { Acha a comunidade onde vive } \\
\text { violenta }\end{array}$ & & & & & $<0,001$ \\
\hline Não & $68(82,0)$ & $49(55,7)$ & $76(60,0)$ & $151(73,6)$ & \\
\hline Sim & $15(18,0)$ & $39(44,3)$ & $51(40,0)$ & $54(26,4)$ & \\
\hline
\end{tabular}

${ }^{a}$ Acende Candeia ( $\left.\mathrm{n}=77\right)$; Matões $(\mathrm{n}=80)$; Parada $(\mathrm{n}=118)$; Pecém $(\mathrm{n}=197)$.

Fonte: Elaborado pelos autores.

ção direta entre percepção de risco, presença altas emissões de poluentes na atmosfera e proximidade dos eventos, ocorreu em Parada.

Assim como em Parada, no estudo sobre diagnóstico participativo socioambiental e de riscos à saúde das comunidades do entorno do Complexo Petroquímico do COMPERJ, a maioria dos participantes $(89 \%$ dos grupos focais e $60 \%$ das entrevistas) identificaram piora na qualidade do $\operatorname{ar}^{16}$. Em estudo transversal realizado 
Tabela 3. Percepções sobre o CIPP, por comunidade. São Gonçalo do Amarante e Caucaia, Ceará, 2018.

\begin{tabular}{|c|c|c|c|c|c|}
\hline \multirow{2}{*}{ Variável } & $\begin{array}{c}\text { Acende Candeia } \\
(n=83)\end{array}$ & $\begin{array}{c}\text { Matões } \\
(\mathbf{n}=\mathbf{8 8})\end{array}$ & $\begin{array}{c}\text { Parada } \\
(\mathbf{n}=127)\end{array}$ & $\begin{array}{c}\text { Pecém } \\
(\mathrm{n}=205)\end{array}$ & \multirow[t]{2}{*}{ p-valor } \\
\hline & n $(\%)$ & n (\%) & $\mathbf{n}(\%)$ & $\mathbf{n}(\%)$ & \\
\hline Considera o CIPP & & & & & $<0,001$ \\
\hline Bom & $39(47,0)$ & $77(87,5)$ & $33(26,0)$ & $173(84,4)$ & \\
\hline Indiferente & $28(33,7)$ & $10(11,4)$ & $43(33,9)$ & $28(13,6)$ & \\
\hline Ruim & $16(19,3)$ & $1(1,1)$ & $51(40,1)$ & $4(2,0)$ & \\
\hline Vida hoje em relação à antes do CIP & & & & & $<0,001$ \\
\hline Melhor & $33(39,8)$ & $36(40,9)$ & $28(22,0)$ & $76(37,1)$ & \\
\hline Igual & $26(31,3)$ & $35(39,7)$ & $52(41,0)$ & $45(21,9)$ & \\
\hline Pior & $18(21,7)$ & $4(4,5)$ & $37(29,1)$ & $13(6,3)$ & \\
\hline Não morava aqui & $6(7,2)$ & $13(14,9)$ & $10(7,9)$ & $71(34,7)$ & \\
\hline Gostaria de mudar de lugar & & & & & 0,029 \\
\hline Sim & $21(25,3)$ & $12(13,6)$ & $40(31,5)$ & $53(25,9)$ & \\
\hline Não & $62(74,7)$ & $76(86,4)$ & $87(68,5)$ & $152(74,1)$ & \\
\hline
\end{tabular}

Fonte: Elaborado pelos autores.

Tabela 4. Caracterização da amostra de acordo com variáveis antropométricas, clínicas e de qualidade de vida, por comunidade. São Gonçalo do Amarante e Caucaia, Ceará, 2018.

\begin{tabular}{|c|c|c|c|c|c|}
\hline \multirow[t]{2}{*}{ Variável } & $\begin{array}{c}\text { Acende } \\
\text { Candeia }(n=83) \\
\end{array}$ & Matões $(n=88)$ & $\begin{array}{c}\text { Parada } \\
(n=127)\end{array}$ & $\begin{array}{l}\text { Pecém } \\
(n=205)\end{array}$ & \multirow[t]{2}{*}{ p-valor } \\
\hline & $\mathbf{n}(\%)$ & n (\%) & n (\%) & n (\%) & \\
\hline SF-36 Percepção geral de saúde & & & & & $0,082^{\mathrm{a}}$ \\
\hline média/DP; mediana & $59,4 / 22,8 ; 62$ & $67,3 / 24,4 ; 72$ & $64,9 / 23,2 ; 67$ & $66,7 / 23,3 ; 72$ & \\
\hline $\begin{array}{l}\text { SF- } 36^{\text {b }} \text { Limitações das } \\
\text { atividades diárias }\end{array}$ & & & & & $0,373^{a}$ \\
\hline média/DP; mediana & $70,2 / 28,9 ; 80$ & $76,2 / 27,9 ; 90$ & $76,7 / 26,7 ; 85$ & $74,3 / 27,3 ; 85$ & \\
\hline $\begin{array}{l}\text { SF-36 Limitações desempenho } \\
\text { em decorrência de problemas } \\
\text { emocionais }\end{array}$ & & & & & $<0,001^{\circ}$ \\
\hline média/DP; mediana & $72,3 / 36,7 ; 100$ & $78,4 / 37,5 ; 100$ & $76,6 / 37,2 ; 100$ & $62,0 / 35,2 ; 66,7$ & \\
\hline $\begin{array}{l}\text { SF- } 36^{\mathrm{b}} \text { Limitações de atividades } \\
\text { sociais }\end{array}$ & & & & & $0,321^{a}$ \\
\hline média/DP; mediana & $79,2 / 25,5 ; 100$ & $84,2 / 23,2 ; 100$ & $85,7 / 24,7 ; 100$ & $82,4 / 27,5 ; 100$ & \\
\hline $\mathrm{IMC}^{\mathrm{c}}$ & & & & & 0,008 \\
\hline Normal & $28(35,4)$ & $28(32,6)$ & $32(26,0)$ & $44(21,8)$ & \\
\hline Sobrepeso & $35(44,3)$ & $37(43,0)$ & $49(39,8)$ & $68(33,7)$ & \\
\hline Obesidade & $16(20,3)$ & $21(24,4)$ & $42(34,2)$ & $90(44,5)$ & \\
\hline Pressão Arterial (PA) & & & & & 0,222 \\
\hline Normal & $42(51,2)$ & $33(37,9)$ & $47(37,0)$ & $80(39,6)$ & \\
\hline Pré-hipertensão & $27(32,9)$ & $34(39,1)$ & $47(37,0)$ & $64(31,7)$ & \\
\hline Hipertensão & $13(15,9)$ & $20(23,0)$ & $33(26,0)$ & $58(28,7)$ & \\
\hline
\end{tabular}

Fonte: Elaborado pelos autores. 
com 240 residentes em localidades no município de Itaboraí-RJ, 37,1\% consideraram a qualidade do ar ruim, sendo diferente a percepção entre as áreas, pois em Porto das Caixas (32,1\%) e Manilha (45\%) fizeram uma avaliação negativa da qualidade do $\operatorname{ar}^{18}$. Estudo realizado em 2006 na área de influência da indústria petroquímica de Guamaré, Rio Grande do Norte, identificou que a ocorrência de sintomas respiratórios em crianças e adolescentes nas comunidades no entorno do pólo, mesmo em baixas concentrações de poluentes atmosféricos, esteve associada a residência na direção preferencial dos ventos ${ }^{20}$.

No estudo que estimou a concentração diária do material particulado fino (MP) 2,5 na atmosfera, em três áreas de influência do CIPP (São Gonçalo do Amarante, Paracuru e Paraipaba e Caucaia), no período de 2006 a 2017, identificou-se o maior pico de concentração na região de São Gonçalo do Amarante, entre os anos de 2011 a 2017 (período de maior consolidação de operação das indústrias), nos meses referentes ao período de seca (setembro a fevereiro) ${ }^{21}$. Esse estudo corrobora com a percepção popular da Comunidade de Parada, pois, apesar das médias anuais das concentrações estimadas, mesmo em São Gonçalo do Amarante, estarem inferiores ao estabelecido pela legislação nacional ${ }^{22}$ nas áreas estudadas, já existem estudos ressaltando a importância que os padrões considerados seguros para a saúde humana sejam revistos ${ }^{23,24}$. O estudo ressalta ainda a importância de avaliar a constituição das partículas finas e sua possível associação a efeitos adversos à saúde da população do entorno do CIPP ${ }^{21}$.

Por fim, Acende Candeia é a comunidade mais diferenciada de todas, estando localizada mais afastada do CIPP, contendo o maior percentual de agricultores $(24,1 \%)$ e aposentados $(25,3 \%)$ e de moradores que relatam morar desde que nasceram $(47 \%)$. Foram também os moradores que mais identificaram a falta de água como um problema $(14,5 \%)$ na comunidade e que identificaram o município como o responsável pelo problema (39\%). Foi a segunda comunidade que pior avaliou o CIPP (ruim - 19,3\%; a vida piorou após o CIPP - 21,7\%). Em relação a qualidade de vida, Acende Candeia foi a comunidade com os piores resultados médios das dimensões percepção geral de saúde $(59,4)$ e limitações das atividades diárias $(70,2)$. Este território é a área mais rural deste estudo, sofrendo os efeitos da seca do Sertão Nordestino, e está na direção da pluma de dispersão dos empreendimentos. Essa realidade talvez reflita na avaliação da qualidade de vida do território.
Em estudo com residentes em área rural, acima de 18 anos, no município de Atibaia-SP, o domínio percepção geral da saúde $(73,1)$, limitações da vida diária $(84,4)$, limitações devido aos problemas emocionais $(83,8)$, limitações sociais $(87,7)$ foram melhores que as médias encontradas em todos os territórios deste estudo, apesar de populações semelhantes ${ }^{25}$. Em estudo com idosos, vinculados a ESF, do município de Cruz Alta-RS ${ }^{26}$, obtiveram os domínios estado geral de saúde $(86,0)$, limitações da vida diária $(87,5)$, limitações de atividades sociais $(86,9)$ superiores em todos os territórios estudados. Os idosos de Cruz Alta só ficaram abaixo da população estudada nas limitações por problemas emocionais $(77,7)$, abaixo somente de Matões $(78,4)$.

$\mathrm{O}$ excesso de peso esteve presente em grandes proporções em todas as comunidades avaliadas. Constata-se, no Pecém, que 78,2\% das pessoas estão acima do peso. Nesta comunidade, a obesidade esteve presente em 44,5\% da amostra, sendo a proporção quando comparada às demais $(\mathrm{p}=0,008)$. De fato, o excesso de peso tem sido considerado um sério problema de saúde pública no globo terrestre. Diversos autores têm encontrado resultados similares ao da presente pesquisa, confirmando nossos achados e reiterando que medidas de intervenção devem ser pensadas para reverter essa situação ${ }^{27,28}$. Já é de conhecimento da literatura que o excesso de peso está diretamente associado a problemas de saúde, como infarto agudo do miocárdio, acidente vascular encefálico, hipertensão arterial sistêmica e diabetes mellitus ${ }^{29}$.

A pressão arterial também é considerada uma variável importante quando se pretende avaliar a saúde da população. Sabe-se que níveis pressóricos aumentados contribuem para desfechos negativos em saúde, tai como acidente vascular encefálico $^{30,31}$. Na Pesquisa Nacional de Saúde ${ }^{32}$, que avaliou a prevalência de hipertensão arterial na população adulta brasileira identificou 22,8\% para hipertensão arterial medida, valor ligeiramente abaixo deste estudo (24,6\%), mas apresentando uma prevalência maior no Pecém $(28,7 \%)$.

Apresentamos aqui um estudo seccional populacional, onde procuramos utilizar técnicas de amostragem e coleta de dados consistentes com este tipo de estudo e entrevistar a totalidade dos moradores nos domicílios, mas isso não foi possível. Nossa amostra é composta, na sua maioria, de mulheres $(69,8 \%)$, sendo $52,4 \%$ com idades entre 30-59 anos e, talvez, nosso resultado não possa ser generalizado para o todo dos territórios estudados. Entretanto, é uma amostra represen- 
tativa das pessoas que mais ficam em casa, provavelmente, mais afetadas pelos problemas locais. Há, ainda, a limitação do desenho de estudo escolhido. Embora o nexo causal fique mais evidente em queixas relacionadas a poluição atmosférica na comunidade de Parada, o mesmo não se pode dizer de variáveis relacionadas a medidas efetivas como IMC e pressão arterial ou medidas de qualidade de vida. Cerca de 35\% dos moradores do Pecém e 15\% dos moradores de Matões não moravam no território antes da instalação do CIPP, indicando um grau de migração para a área pelo complexo sem um estudo prévio da saúde destas populações.

Essa realidade da amostra do estudo, dificulta estabelecer relações entre atividade profissional e as comunidades, tendo sido encontrado o maior percentual de trabalhadores do $\operatorname{Lar}(31,2 \%)$, aposentados $(16,3 \%)$ e trabalhadores sem atividade no momento da pesquisa $(10,3 \%)$, sendo a comunidade do Pecém o maior percentual sem atividade $(13,2 \%)$. Destaca-se o baixo percentual de colaboradores do CIPP em todas as comunidades, não podendo afirmar se o CIPP emprega pouco os seus moradores ou se estes estavam trabalhando no momento da coleta de dados. Por fim, algumas inconsistências foram identificadas nas respostas, como a contradição entre a sensação de insegurança entre os moradores do Pecém e o baixo percentual $(26,4 \%)$ de identificação do território como violento, além da aparente discrepância entre o número de aposentados relatados nos benefícios $(25,3 \%)$ e a identificação de aposentado na atividade profissional (15,7\%), o que talvez possa ser explicado se adicionarmos os $10,9 \%$ das respostas de "sem atividades".

Nosso estudo teve seu cálculo amostral baseado nos cadastros da Estratégia de Saúde da Família local, o que torna possível um diagnóstico e planejamento local das equipes da ESF, ressaltando que essas comunidades possuem aspectos diferentes de outras comunidades rurais do Ceará. Os resultados foram apresentados em reuniões com as equipes da ESF, gestores, residentes e com a população local. Essa parceria com a ESF foi fundamental uma vez que são os profissionais de saúde da estratégia que são responsáveis pelo acompanhamento clínico da população local impactada pelos empreendimentos, sendo eles em melhor condição de detectar precocemente tais determinantes.

Reforça-se a importância de estudos prospectivos que avaliem as percepções, condições de vida e saúde de moradores do entorno do CIPP, pois estes serão os primeiros a sentir os impactos na saúde. Silveira e Araújo Neto ${ }^{33}$ evidenciam a importância da participação do setor saúde nos processos de licenciamento ambiental de grandes empreendimentos, pois este é o primeiro a sentir as consequências dos impactos ambientais na população do entorno.

Embora próximas, as características das quatro comunidades diferem bastante e parecem estar relacionadas à localização dos territórios em relação ao CIPP. Nas comunidades de Pecém e Matões, mais urbanizadas, de maior população e mais próximas do terminal Portuário, houve uma maior frequência de relatos semelhantes aos grandes centros urbanos, tais como, violência, prostituição e alto consumo de álcool e drogas ilícitas. Já a comunidade mais afetada pela poluição por material particulado (Parada) por estar na direção da pluma de dispersão da siderúrgica do CIPP, apresentou a visão mais negativa do CIPP. Dados de qualidade de vida e informações básicas antropométricas de saúde, indicam um grau considerável de comprometimento da saúde da população local. A comunidade mais afastada (Acende Candeia) foi a que mais apresentou uma atividade de trabalho mais relacionada com a agricultura, atividade predominante, juntamente com a pesca, antes da construção do CIPP. Ao contrário do esperado, encontramos poucas pessoas em todas as comunidades com atividades laborais diretamente relacionadas ao CIPP, podendo estar relacionado ao estágio atual das atividades no complexo mais voltadas para aquelas que demandem mão de obra mais especializada.

\section{Conclusão}

Embora o desenho de estudo utilizado não permita que se façam afirmações causais, essa pesquisa apresentou indícios de impacto na saúde e qualidade de vida das populações locais sem maiores benefícios diretos no trabalho/renda resultante de empregabilidade no complexo. Os problemas relacionados às limitações do estudo reforçam, ainda, a importância de avaliações de impacto à saúde previamente à instalação de grandes empreendimentos para que futuros impactos possam ser mitigados e para que monitoramentos futuros possam mostrar relações causais entre indicadores de saúde e presença dos empreendimentos. 


\section{Colaboradores}

SAS Nuto, EB Barreira Filho, BFA Oliveira, RWJF Freitas, LO Couto, LSV Jacobson, SS Hacon, ARS Périssé, participaram da concepção, planejamento, coleta de dados, análise e interpretação dos dados; contribuíram na elaboração, revisão e aprovação da versão final do artigo.

\section{Agradecimentos}

Para o desenvolvimento desta pesquisa agradecemos ao financiamento do Ministério da Saúde e a parceria da Secretaria da Saúde do Estado do Ceará e Secretarias Municipais de Saúde de São Gonçalo do Amarante e de Caucaia.

\section{Referências}

1. Bezerra MGV. Do canto das nambus ao barulho do trem: transformações no modo de vida e na saúde na comunidade de Bolso no Complexo Industrial e Portuário do Pecém-CE [dissertação]. Fortaleza: Universidade Federal do Ceará; 2010.

2. Assembleia Legislativa do Estado do Ceará. Conselho de altos estudos e assuntos estratégicos. Cenário atual do complexo industrial e portuário do Pecém. Fortaleza: INESP; 2013.

3. Bezerra MGV, Rigotto RM, Pessoa VM, Silva FVE. Implicações do desenvolvimento econômico no trabalho, ambiente e saúde em comunidades portuárias no Ceará, Brasil. Cien Saude Colet 2014; 19(10):40234030 .

4. Rigotto RM. Desenvolvimento, ambiente e saúde: im plicações da (des)localização industrial. Rio de Janeiro: Fiocruz; 2008.

5. Gurgel AM, Medeiros ACLV, Alves PC, Silva JM, Gurgel IGD, Augusto LGS. Framework dos cenários de risco no contexto da implantação de uma refinaria de petróleo em Pernambuco. Cien Saude Colet 2009; 14(6):2027-2038.

6. Fase-Ettern. Relatório síntese: Projeto de avaliação de equidade ambiental como instrumento de democratização dos procedimentos de avaliação de impacto de projetos de desenvolvimento. Rio de Janeiro: Fase-Ettern; 2011.

7. Burger J, Myers O, Boring CS, Dixon C, Jeitner JC, Leonard J, Lord C, McMahon M, Ramos R, Shukla S, Gochfeld M. Perceptual indicators of environmental health, future land use, and stewardship. Environ Monit Assess 2003; 89(3):285-303.

8. Candeias NMF, Abujamra AMD, Oliveira JT. Percepção de trabalhadores metalúrgicos sobre problemas de saúde e riscos ambientais. Rev Esc Enferm USP 1998; 32(3):231-246.

9. Moniz MA. Amianto, perigo e invisibilidade: percepção de riscos ambientais e à saúde de moradores de município de Bom Jesus da Serra/Bahia [dissertação]. Rio de Janeiro: Escola Nacional de Saúde Pública Sérgio Arouca; 2010.

10. Di Giulio GM, Vasconcellos MP, Günther WMR, Ribeiro H, Assunção JV. Percepção de risco: um campo de interesse para a interface ambiente, saúde e sustentabilidade. Saude Soc 2015; 24(4):1217-1231.

11. Ciconelli RM, Ferraz MB, Santos W, Meinão I, Quaresma MR. Tradução para a língua portuguesa e validação do questionário genérico de avaliação de qualidade de vida SF-36 (Brasil SF-36). Rev Bras Reumatol 1999; 39(3):143-150.

12. World Health Organization (WHO). Guia de intervenção mhGAP - versão 2.0 [Internet]. [acessado 2020 mar 23]. Disponível em: https://www.who.int/ publications/i/item/mhgap-intervention-guide--version-2.0.

13. Open Data Kit [Internet]. [acessado 2020 mar 23]. Disponível em: h8p://code.google.com/p/open $<$ data $<$ kit.

14. Departamento de Terapia Ocupacional (UFPR). Cálculo dos escores do questionário de qualidade de vida [Internet]. [acessado 2020 mar 23]. Disponível em: https://toneurologiaufpr.files.wordpress.com/2013/03/ questionc3a1rio-de-qualidade-de-vida-sf-36-cc3allculo-escores.pdf. 
15. Osterno CAN. Utilização de drogas lícitas e ilícitas em uma população de adolescentes escolares do distrito de Pecém-CE [dissertação]. Fortaleza: Universidade de Fortaleza; 2019.

16. Moniz MA, Sabóia VM, Carmo CN, Hacon SS. Diagnóstico participativo socioambiental e de riscos à saúde das comunidades do entorno do Complexo Petroquímico do Rio de Janeiro, Brasil. Cien Saude Colet 2017; 22(11):3793-3805.

17. Moniz MA, Pereira JM, Dias RM. Impactos psicossociais do contexto de construção do complexo petroquímico do Rio de Janeiro. Trabalho Educ Saude 2017; 15(2):439-451.

18. Moniz MA, Carmo CN, Hacon SS. Percepção da qualidade ambiental de localidades próximas ao Complexo Petroquímico do Rio de Janeiro, Brasil. Cien Saude Colet 2016; 21(6):1871-1878.

19. Brody SD, Zahran S, Vedlitz A, Grover H. Examining the relationship between physical vulnerability and public perceptions of global climate change in the United States. Environment Behavior 2008; 40(1):7295.

20. Moraes ACL, Ignotti E, Netto PA, Jacobson LSV, Castro H, Hacon SS. Wheezing in children and adolescents living next to a petrochemical plant in Rio Grande do Norte, Brazil. J Pediatr 2010; 86(4):337344.

21. Couto LO, Nuto SAS, Hacon SS, Gioda A, Sousa FW, Barreira Filho EB, Gonçalves KS, Périssé ARS. Estimativa da concentração média diária de material particulado fino na região do Complexo Industrial e Portuário do Pecém, Ceará, Brasil. Cad Saude Publica 2020; 36(7):e00177719.

22. Brasil. Conselho Nacional do Meio Ambiente (CONAMA). Resolução no 491, de 19 de novembro de 2018. Dispõe sobre padrões de qualidade do ar. Diário Oficial da União 2018; 21 nov.

23. Shi L, Zanobetti A, Kloog I, Coull BA, Koutrakis P, Melly SJ, Schwartz JD. Low-concentration PM2.5 and mortality: estimating acute and chronic effects in a population-based study. Environ Health Perspect 2016; 124:46-52.

24. Nascimento AP, Santos JM, Mill JG, Souza JB, Reis Júnior NC, Reisen VA. Associação entre concentração de partículas finas na atmosfera e doenças respiratórias agudas em crianças. Rev Saude Publica 2017; 51:3.

25. Lima PJP, Oliveira HB. Aspectos de saúde e qualidade de vida de residentes em comunidades rurais. Rev Baiana Saude Publica 2014; 38(4):913-930.
26. Wanderley BS, Reinaldo G, Voloski FRS, Monteiro $\mathrm{MB}$, Elsner V. Efeito do sobrepeso e da obesidade sobre função pulmonar e qualidade de vida de idosos vinculados ao programa estratégia da saúde da Família no município de Cruz Alta-RS. Estud Interdiscipl Envelhec 2018; 23(1):61-74.

27. Melo SPSC, Cesse EAP, Lira PIC, Ferreira LCCN, Ris$\sin$ A, Batista Filho M. Sobrepeso, obesidade e fatores associados aos adultos em uma área urbana carente do Nordeste Brasileiro. Rev Bras Epidemiol 2020; 23:E200036.

28. Brebal KMM, Silveira JAC, Menezes RCE, Epifânio SBO, Marinho PM, Longo-Silva G. Ganho de peso e mudança do estado nutricional de brasileiros após os 20 anos de idade: uma análise de série temporal (2006-2012). Rev Bras Epidemiol 2020; 23:E200045.

29. Sousa NA, Lima JS, Teixeira TC, Linhares CB, Montes JVL, Marques JVS. Fatores de risco e complicações em diabéticos/hipertensos cadastrados no hiperdia. Sanare 2019; 18(1):31-39.

30. Haito SM, Moreira KFA, Freitas JLG, Souza RA, Farias ES. Risco cardiovascular em hipertensos cadastrados em uma unidade de saúde no Norte do Brasil. Rev Bras Promoç Saude 2020; 33:10400.

31. Vaz DWN, Evangelista HI, Pontes LC, Silva JB, Rezende RWS, Acatauassú LP. Perfil epidemiológico do Acidente Vascular Cerebral no Estado do Amapá, Brasil. Res Soc Development 2020; 9(8):e938986642.

32. Malta DC, Gonçalves RPF, Machado IE, Freitas MIF, Azeredo C, Szwarcwald CL. Prevalência da hipertensão arterial segundo diferentes critérios diagnósticos, Pesquisa Nacional de Saúde. Rev Bras Epidemiol 2018; 21(Sup. 1):E180021.

33. Silveira M, Araújo Neto MD. Licenciamento ambiental de grandes empreendimentos: conexão possível entre saúde e meio ambiente. Cien Saude Colet 2014; 19(9):3829-3838.

Artigo apresentado em 21/07/2020

Aprovado em 22/02/2021

Versão final apresentada em 24/02/2021

Editores-chefes: Romeu Gomes, Antônio Augusto Moura da Silva 